\title{
RISK MANAGEMENT PRACTICES IN PROJECT ACTIVITIES AT NORTH KUWAIT OIL FIELDS
}

\author{
AHMED M. QABAZARD \\ Health, Safety \& Environment (North Kuwait) Team, Kuwait Oil Company, Kuwait
}

\begin{abstract}
Risk management is a concept which has become very popular in a number of business organizations. Many companies often establish a risk management procedure in their projects for improving the performance and increasing profits. The project construction industry is highly risk prone, with complex and dynamic environments which create an atmosphere of high uncertainties and potential hazards. The project sector is vulnerable to various technical, socio-political and business risks including HSE (health, safety and environment). The track records to cope with these risks have not been very good in the construction industry. As a result, the people working in the project industries bear various failures, such as failure to abide by quality and operational requirements, exceeding cost and uncertain delays in project completion including HSE obligations. The risk based approach or risk management is an important process of project activities which consist of the identification of risks, appropriate assessment, responses with a suitable method for handling risks and then developing strategies to mitigate those risks in compliance with the regulatory objectives and the company's risk management expectations. This paper covers the concepts of HSE risk management through various risk evaluations, project challenges and effective risk mitigation/reduction practices being followed at Kuwait Oil Company (KOC) - North Kuwait (NK) oil fields - for the one stop solution for all types of hazards and enhancing HSE compliance during any construction project lifecycle. The risk based approach consists of both proactive and reactive control and corrective action that could be applied according to the nature, severity and immediacy of the risk posed, which enables one to be flexible, adaptive and focused to the project HSE demands and ongoing changes in the project plan without affecting contractual HSE obligation. With the above approach, there is a visible improvement in HSE compliance at contractors' worksites at NK oil fields, which signifies the company's HSE commitment in its business process.

Keywords: risk management, project risk.
\end{abstract}

\section{INTRODUCTION}

The role of contractors in industrial development, especially in oils sectors, are very vital. Industrial sector cannot develop with the current growing demand without the assistance of proper contracting agencies. The contractors are key players in the sustainable oil sector projects from predesign and design, through construction and project closeout. They also provide immense support in operation and maintenance of our production facilities. However, in this context a question always arises in our mind that, how do the project/ construction risks are managed effectively? There are high potential risks involved in the project activities including key challenges and their implications.

In order to address the above challenges, we follow some of the monitoring practices as a part of project risk management to ensure HSEMS (health, safety and environment management system) compliance [1] at contractors' worksites through regular site inspections, site verification visits, mobile equipment integrity surveys, audits, on- site awareness, campaigns, etc. These monitoring practices are not only to ensure site compliance, but also provide the opportunities for continual risk improvements.

\section{NECESSITY}

Globally it is acknowledged that an improved HSE compliance implementation through riskbased approach has direct and strategic bearings on functional and operating project HSE 
performance standards [2] of various contractors. Project accomplishments involve various intended activities of pre-design, design, review, construction, testing, inspection, commissioning, stabilization process, etc. to be carried out by multiple agencies within the specified period. Sometime this involves surprises (unplanned activities) due to various reasons. Because of constraints of time and resources, there is a possibility of bypassing the obligatory/regulatory HSE compliance leading to enhancement of risk level to undesired/ unacceptable level. Hence, the established and effective risk management practices will ensure proper identification of risk, its' assessment and suitable control/mitigation measures in the method for handling risks and then develop strategies to mitigate those risks in compliance project activities.

\section{OBJECTIVE}

The purpose of this risk management practice is to evaluate the HSE performance of North Kuwait-based contractors [2] by reviewing their worksite HSE compliance level, policies, statistical performance, adherence to standards, etc. The areas of improvement and positive observations identified through this process are used as a basis for the risk scrutiny and resultant recommendations, in order to improve upon HSEMS procedural compliance [3] at contractors' worksites. The objectives are summarized as follows:

- HSE surveillance (third-eye view) at contractors' worksites;

- Timely HSE support;

- Proper risk assessments of all critical tasks;

- Recording the lessons learnt for future.

The long-term intent of the risk management practices is to build project findings to document best practices, lessons learned, and examples of various documents to benefit $\mathrm{KOC}$ and the contractors in terms of benchmarking, evaluation and enhancing HSEMS performances.

\section{CHALLENGES OF PROJECT RISK MANAGEMENT}

Project construction activities are risk level with complexities, which pose environment of high uncertainties and potential hazards. Each project task is critical and have its own importance and intricacies in handling the activities. Some of the inherent challenges faced in managing HSE compliance during the project execution are as follows:

- Intense competition for available work;

- Multiple critical activities;

- Diverse agencies;

- Diminished work supervision;

- Migrant/new work force in changing environment;

- Management of waste generated out of construction;

- Project schedule goals and targets for safety, quality and cost;

- Multiple agencies with combination of experienced and novice workforce having different age groups poses greater risk and challenge in execution of safe work practices;

- A high number of litigation cases as well as disputes.

\section{METHODOLOGY}

The above risk management process is unique and different from various conventional methods, which focuses not only on level of implementation but also emphasis on deviations 
observed during the course of verification. The following steps are involved in the above process.

5.1 Developing a comprehensive analytical tool/framework utilizing various inspection databases

\subsubsection{HSE inspections at project worksites}

There are comprehensive, planned and periodic inspection schedules prepared for each project sites to provide accurate and objective feedback to management and the adequacy, effectiveness, and efficiency of contractors' HSE programs, compliance and activities compared to applicable requirements. The objectives of the inspection process (Fig. 1) are to evaluate the progress of achieving HSE targets, maintaining regulatory compliance, and effectively utilizing the KOC health safety and environment management system [1] including reporting deficiencies in meeting KOC HSE requirements and commitments. Results of the evaluation of the collected inspection evidence compared with the inspection criteria or the results of an inspection. Findings can indicate conformity or nonconformity with audit criteria, good practices and/or opportunities for improvement.

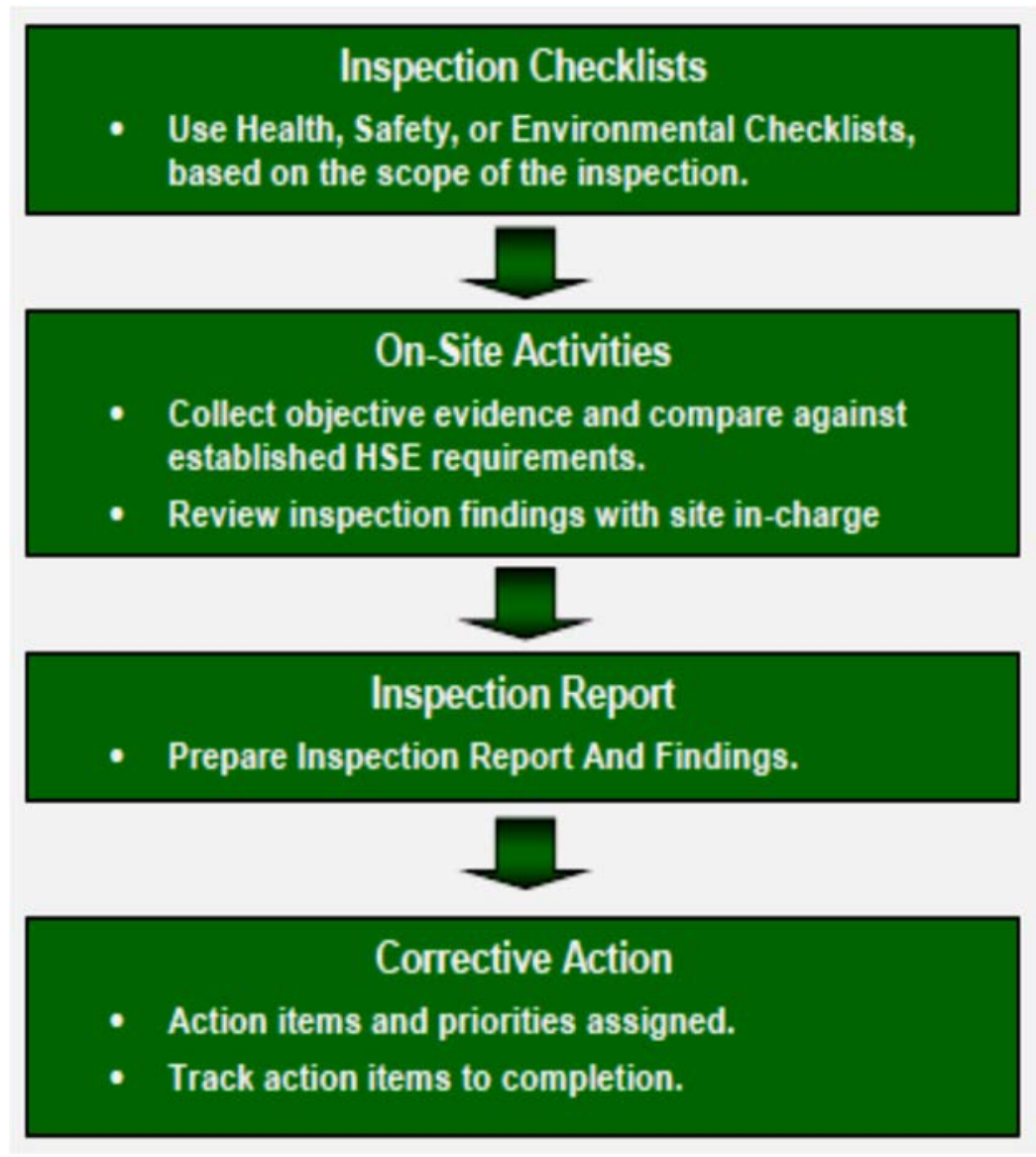

Figure 1: HSE inspection process. 
5.1.2 Observations from site verification visits (SVV's)

Site verification visits are the planned visits to the contractors' worksites (typical project worksite as shown in Fig. 2) by either the group or the individual and verify the level of HSE compliance at site and any deviations observed shall be discussed at the visit location/spot and highlighted to the respective controlling teams the project. Usually these SVV are conducted focusing on the HSE themes identified specifically for the month. The management team shall carry out site visit for the specific location indicated in the visit schedule. The main objective of this site visit is to enhance HSE commitment and leadership demonstration at worksite. The observation during site verification visit shall be focused to issues enlisted in the specific checklist as applicable. The observation made by the visitor (management personnel) during such visit shall be reported online through MyHSSE [4].

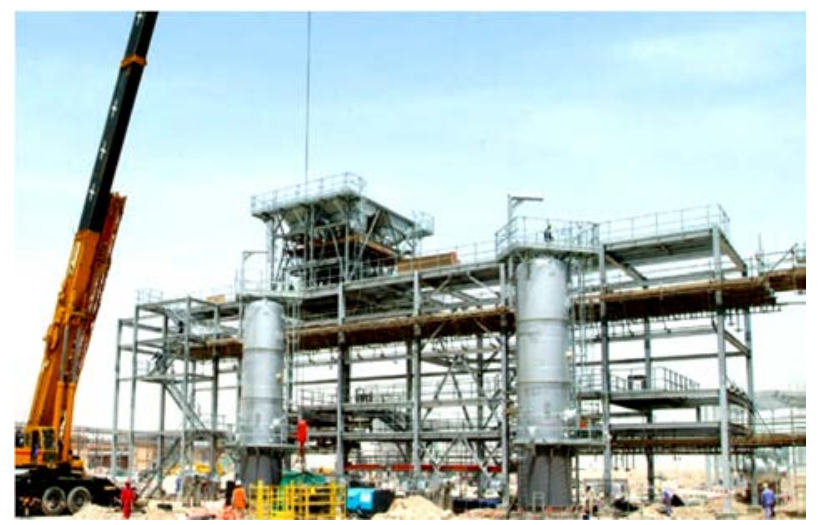

Figure 2: Typical project worksite.

\subsubsection{HSE inspections at project site offices}

It is similar process like Inspection at project sites. However, it emphases on the compliance requirements as per KOC standard for portacabins especially on material of construction, electrical installations/fixtures and fire protection arrangements considering the fire hazard potentials.

5.1.4 Other HSE leading and lagging indicator performance from MyHSSE [4] data Making a HSE program that includes the right elements for assessing HSE performance, like leading and lagging indicators, is a critical step in boosting overall HSE performance. Leading and lagging indicators have a valuable effect on measuring HSE performance. Lagging indicators are reactive in nature. They measure the effectiveness of a safety program after the facts. Typical lagging indicators include the number of incidents, injuries, LTIs (lost time injuries), incident frequency rates, etc. Whereas, leading indicators are proactive in nature. These indicators focus on the future. They target events used to drive and measure activities designed to prevent and control behavior and injuries. Typical leading indicators include the safety training, audit/inspections conducted, etc. Both leading and lagging indicators are relevant to workplace safety, especially at project sites and worth measuring. They present important aspects of an overall HSE management system that drives all to a zero-incident at worksite. All relevant data are entered and logged appropriately in a system-based programme MyHSSE [4] specially design to record and track leading/ lagging indicators. 
5.1.5 Tracking of action items in the project risk registers

The main purpose of the preparing the project risk register to identify occupational safety hazards, assess the risks arising from project activities and ensure its proper management in order to comply with legal and regulatory requirements, company HSSE policy [5] and occupation prerequisites. The entire process of preparation/review of risk registers is conducted through series of workshop sessions involving experienced team of multidisciplinary experts from various teams, who brainstorms on each risk aspects pertaining to scope of project work/activity and accordingly ensure that the identified risks are assessed, prioritized and controlled to eliminate the hazards or reduce the risks to acceptable levels. The typical flow-diagram of risk assessment and management process is shown in Fig. 3.

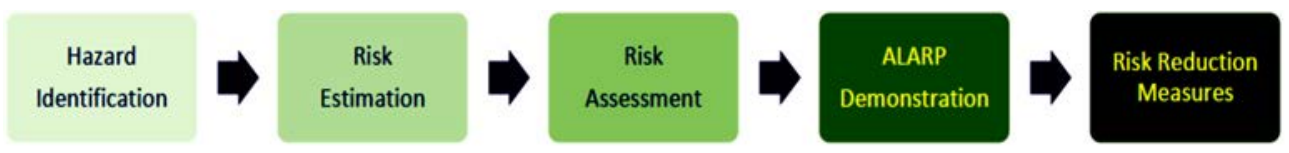

Figure 3: Risk assessment and management process.

5.2 Evaluating HSEMS procedural/standards implementation and compliances based on the risk level

All HSE observations/deviations made at work sites and site offices of all KOC North Kuwait contractors are critically assessed based on the company's risk rating matrix (Fig. 4) as prescribed in KOC HSE management system (HSEMS) procedures [3] and categorized/listed as low (acceptable), medium (tolerable), high (intolerable). The tracking/monitoring of those listed observations are carried out appropriately through a task force team (TFT).

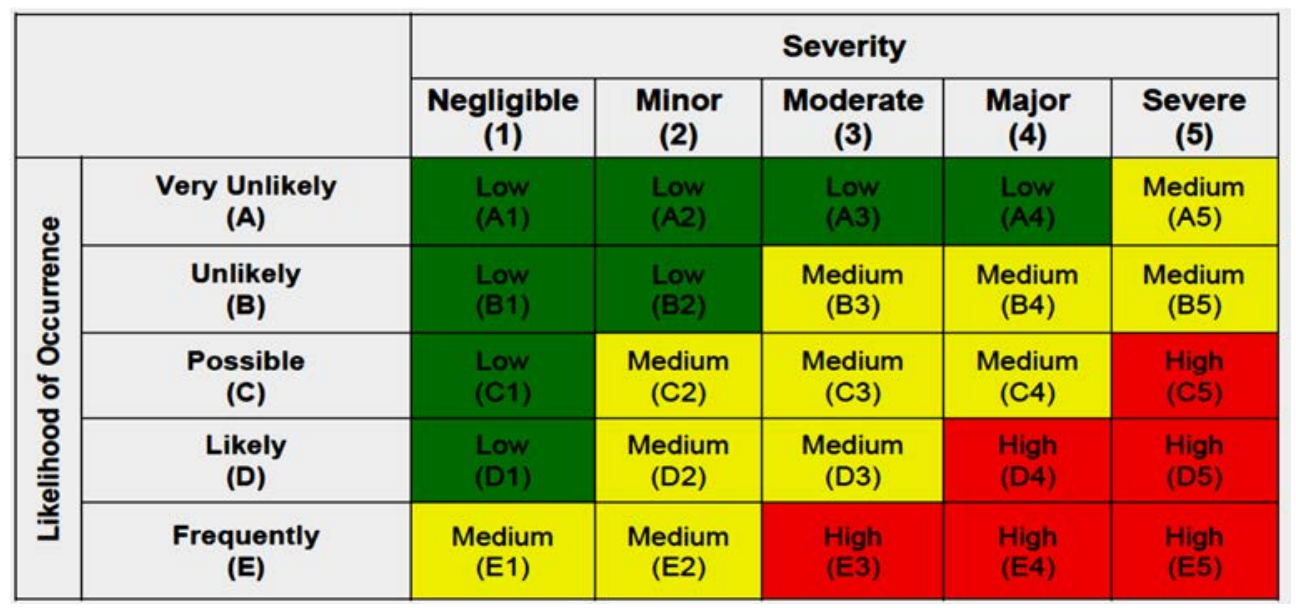

Figure 4: Risk rating matrix. 
5.3 Identifying and analyzing the positive observations/compliance on KOC HSEMS procedures/standards [3]

Ensuring compliance to HSE procedure/standard and maintaining a safe workplace will reduce the risk of work-related illnesses and injuries, etc. The HSE adherence and good safety practices at worksites are also recorded and provided with equal weightage while comparing the overall HSE performance assessment.

5.4 Identification and analysis of critical risk-based gaps/non-compliance of HSEMS procedures/standards [3]

5.5 Sharing results/contractor performance status with respective project controlling teams to improve contractor performance [2]

5.6 Deliberating on performance status with respective contractors during monthly HSE meetings

5.7 Close follow-up with the respective contractors through their project controlling teams for tracking/pursuing and early closure of deviations/ HSE observations made during the surveillance process/system

\section{HSE FOCUS/EMPHASIS}

Considering the complexities of the various project activities, other priorities and management expectations during project job execution, more emphasis/importance have been given on the following HSE priority areas.

\subsection{Project HSE plan/job execution plans}

It is an essential project HSE document that ensures the requirements and expectations of the KOC HSSE policy [5], KOC strategic objectives, regulatory compliance, recommendations of risk insurers, risks and impacts associated with the project, key activities, roles, and responsibilities etc. are addressed adequately and appropriately. This document also explains the relevant project risks are identified and assessed using appropriate tools including mitigation through application of risk management techniques. This document is also reviewed and revised as per the various stages of the project including the project scope.

\subsection{Hazard management and communication}

The goal of the hazard management and communication is to ensure the cost-effective, integrated actions that will reduce or prevent potential risks to all stakeholders.

\subsection{HSE awareness and campaigns to elevate the workers' awareness level}

The HSE awareness and campaign is an effective way to spread a safety-related message to employees/workers. It is a structured course of action to achieve a specific goal of shaping the behavior of the intended target audience and providing practical solutions to HSE issues that have been identified. 


\subsection{HSE surveillance to find the deviations}

HSE surveillance is to be more vigilant for safety event detection (third eye view) at various workplaces/worksites revealing HSE deviations/unsafe practices. The objective of the HSE surveillance process is to ensure greater risk reduction.

\subsection{Audit and compliance to HSE management system [1]}

HSEMS audit is a comprehensive review of company's adherence to HSEMS [1], [3] procedures and standards. Audit reports evaluate the strength and thoroughness of compliance preparations, HSSE (health, safety, security and environment) policy [5].

\subsection{HSE reinforcement/facilitation (assistance)}

The main objective of above is to ensure provide all necessary HSE support and assistance (as applicable and required) to the workforce working under various risks to mitigate the risk level.

\subsection{Support to critical/special project activities}

Providing HSE support for critical and special project activities, which need coordinated review and risk assessment by multi-disciplinary team.

\section{RISK MANAGEMENT PRACTICES}

Project HSE risk management encompasses the assessment and mitigation of project HSE risks. The objective of the above is to assess the risks associated with identified hazards and to develop and implement effective and appropriate mitigations. Project HSE risk management is therefore a key component of the process for both the company and the contractor (service provider). Project HSE risks are conceptually assessed as low, medium, or high (acceptable, tolerable, or intolerable) (Fig. 4). Risks assessed as initially falling in high (intolerable) region are unacceptable under any circumstances. The probability and/or severity of the consequences of the hazards are of such a magnitude, and the damaging potential of the hazard poses such a threat to safety, that immediate mitigation action is required.

Bearing above HSE focal areas (as stated in Section 6) in mind, HSE-NK team has established and following multi-functional proactive measures (as given below) focusing primarily on enhancing the awareness and compliance level at all project worksites ensuring HSE visibility, as a part of risk management practices under accident prevention program at North Kuwait Directorate.

\subsection{Project HSE plan}

Preparation of appropriate project HSE plan ensuring the requirements and expectations of the KOC HSE policy, KOC strategic objectives, regulatory compliance, recommendations of risk insurers, etc. are all addressed. Also ensuring the potential health, occupational and process safety hazards/environmental aspects and corresponding risks and impacts associated with the project are identified and assessed using appropriate tools and are mitigated through application of risk management techniques. The above plan identifies the key activities, roles, and responsibilities that will meet the HSE goals at the various stages of the project. The 
same plan also serves as input, at the early stages of the project, for estimation of the time and resources (monetary and human) required for achieving the project HSE objectives.

\subsection{Project execution HSE plan and work method statements}

Subsequently, respective contractors develop their project execution HSE plan that contains detailed HSE plan and programs that describe how the contractor will meet the company's HSE requirements and will manage the identified HSE risks while performing the applicable activities in the project execution stage that generally will include detailed engineering and design, construction and commissioning. This includes the method statements for various critical activities to address all pertinent HSE aspects during project job execution.

\subsection{Hazard management plan}

Hazard Management comprises of three following parts:

- Identification/recognition;

- Assessment and analysis;

- CAPA (corrective and preventive action).

Through walkthrough and site visit, inspections conducted and identified non-compliance and sub-standard practices. Situations or conditions leading to serious consequences and prone to injuries are brought into considerations. Communicating inherent potential hazards in various project critical activities to the concerned worksite supervisors/workers involved in the job, is one of the effective ways of managing hazards and thus preventing incidents at worksite. HSE-NK team takes special consideration on the above and ensured about the proper hazard identification, control through assessment and communication to the concerned workers through toolbox talk, Supervisor briefing. Surprised checks are being made randomly at some of the worksites having hazardous activities to ensure compliance. Suitable hazard briefings provided to available workforce wherever it was needed/necessary.

\subsection{HSE hazard communication}

Internal HSE hazard communication and management processes are focused on maintaining open lines of communication within and between project operational and support groups. Regularly scheduled meetings at all levels of the organization allow two-way dialogue to improve communication on HSE matters. In addition to regular personal communication between KOC (controlling teams) and the contractors' workforce, KOC maintains a number of communication tools and reference sources to provide employees with on-demand HSE information.

\subsection{Exclusive HSE awareness}

Annual program on "Onsite HSE awareness" sessions exclusively for project contractors' personnel at their worksites on monthly basis covering relevant/applicable HSE topics (namely, working at height, excavation, mobile equipment, work permit/JSA, confined space, electrical safety, driving safety, etc.) covering all high priority/project critical activities apart from theme-based campaigns. Last year more than 4,000 contractors' workers were covered under onsite HSE awareness programme at various levels. This has ensured more clarity on the procedures and effective compliance. 


\subsection{Sharing HSE best practices/lessons learned}

Communicating HSE best practices, lessons learned, safety flashes/messages, HSE circulars, etc. regularly amongst all project contractors through various forums including publishing theme based HSE Flash in company's portal, screen saver, HSE meetings, etc. with an objective of enhancing HSE awareness as continual process.

\subsection{Special HSE monitoring}

Physical examinations and surveys at worksites are being conducted randomly especially for the critical activities, namely, confined space entries, heavy lifting/shifting activities, etc. In the above process, field compliance audits are also carried out considering the potential hazard identification and suitable preventive/protective measures taken including the proper permit issuance as per the applicable procedures. Immediate corrective measures being taken wherever deviations are noticed.

\subsection{Theme-based audits}

Conducting special audits on job-specific activities (namely, permit to work, excavation, etc.) to evaluate the compliance level in order to improve the contractors' and sub-contractors' HSE performance [2].

\section{RESULTS GAINED AND ADVANTAGES}

Addressing HSE issues with a proactive risk-based approach during project execution activities, it not only ensures compliance as per KOC requirements, but also offers significant opportunities to improve HSE performance. There has been significant improvement in the HSE performance of North Kuwait Directorate by following the above HSE best practices in managing project risks at various worksites. Year wise comparison of HSE lagging indicators of NK areas is graphically given in Fig. 5.

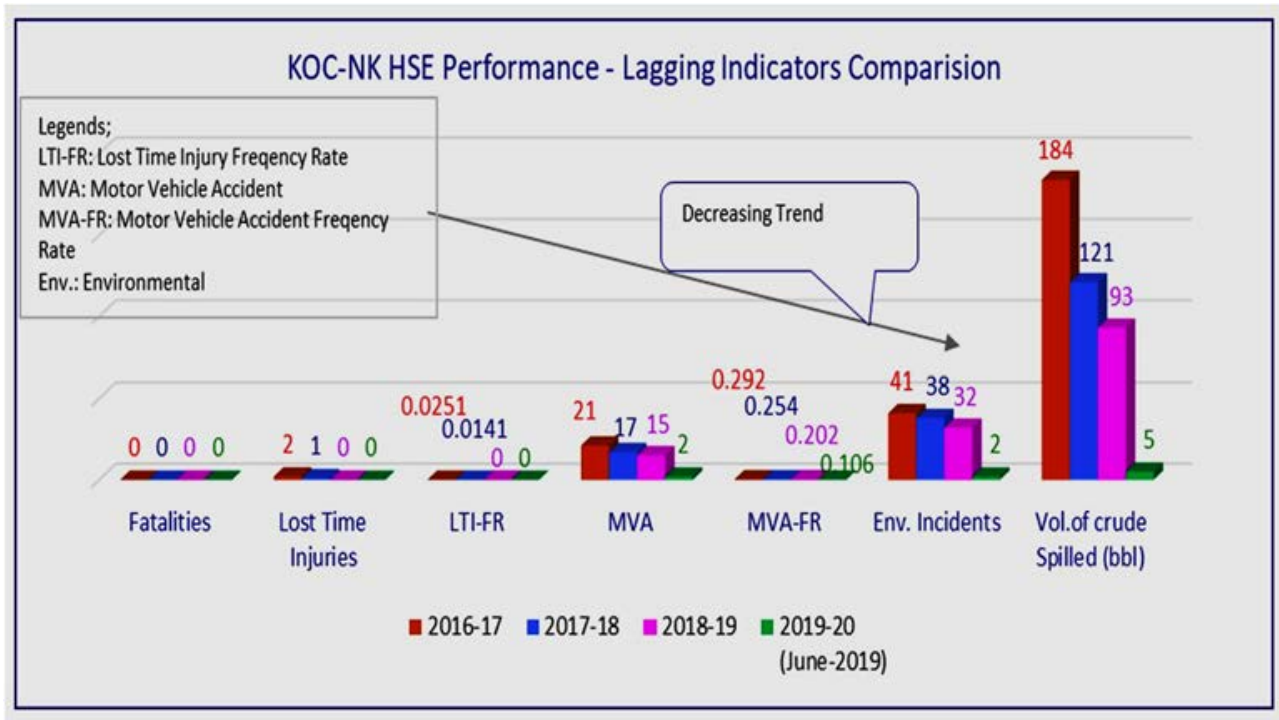

Figure 5: HSE lagging indicators comparison. 
Some of the benefits are as follows:

- Zero lost workday cases (of contractors) in NK Fields since 19th April 2017.

- Provided a mechanism for risk-based approach on contractors HSE compliance to the controlling teams and contractors management.

- Performance of NK contractors is benchmarked, which is utilized to enhance their Performance compliances.

- Benchmarking encourages healthy competition amongst NK contractors to strive for better HSE performance.

- Positive field HSE compliances has increased substantially, which resulted in positive HSE statistics for NK fields.

- Provides a platform for the controlling team to effectively monitor contractor HSE performance compliance level.

- Effective tool for competent workforce hiring, promotions, work assignments, rewards/penalties of contractors.

- Provides a structured approach focusing on contractors' strategic performance objectives.

\section{CONCLUSION}

"Risk management practices" followed during project execution activities is one of the very important approaches for ensuring incident free activities and improved HSE performance. The compliance gaps and positive observations identified through this process are used as a basis for the analysis and subsequent recommendations, in order to improve upon HSEMS procedural compliance. It is proved to be an effective tool for monitoring and management of critical high risks posed by various activities using systematic and structured approach. However, maintaining the consistency in the risk-based approach and supporting its activities is not an easy task. Hence, continuous involvement and deliberations of the team-members with appropriate logistic supports rendered by the HSE-NK leadership is very much essential and vital in order to manage the critical risks in the desired manner.

With the relentless efforts rendered by the HSE-NK team, there are visible improvements in HSE compliance including hassles-free and incident-free activities during entire project job execution period at various project sites at North Kuwait Directorate. The long-term intent is to build project findings to document best practices, lessons learned, and examples of various documents to benefit $\mathrm{KOC}$ and the contractors in terms of benchmarking, evaluation and enhancing HSEMS performances. This kind of initiative clearly signify organization's HSE commitment.

\section{REFERENCES}

[1] KOC, HSE Management System Guide, A framework for company's HSE Management System, KOC internal document.

[2] KOC, HSEMS Performance Reports of Contractors, HSE performance expectations during job execution at KOC, KOC internal document.

[3] KOC, HSE Management System (HSEMS) Procedures, A management tool to achieve the Company's HSE commitment, KOC internal document.

[4] KOC, "MyHSSE" Data, A system based software application for managing all relevant HSE performance records.

[5] KOC, HSSE Policy, Company's philosophy and commitment towards Health, Safety, Security \& Environment, KOC internal document. 